the Newcastle physicians found the converse. Only in the Newcastle study did an initially high serum cholesterol point to an increased risk of infarction during the trial. More reliable information might have emerged if the results of the two studies could have been pooled, but this was not possible because of differences in patient selection. It is also unfortunate that in the Scottish trial the control and treated patients differed from each other with respect to cholesterol level and blood pressure. But it is difficult to see why the presence or absence of angina should influence the usefulness of clofibrate, and, as seems possible from the Newcastle study, it is surprising to find that the benefit from clofibrate might not be related to its lipid-lowering action.

L. R. Krasno and G. J. Kidera, from San Francisco, have now reported their findings 5 in 1,068 men, of whom 67 had ischaemic heart disease, followed up for 62 months. The patients were randomly allocated according to age, blood pressure, weight, smoking habits, lipid levels, and incidence of heart disease. Five hundred and eighteen received clofibrate throughout the study. The other 550 were untreated for 39 months and then received clofibrate for the final 23 months. In the first period the infarction rate fell in clofibrate-treated patients, with and without previous heart disease. The patients treated only in the second period showed a significant reduction in incidence of infarction in that period. In this study clofibrate had no influence on mortality. Cholesterol and triglyceride levels were measured, and $62 \%$ and $70 \%$ of treated patients with abnormal levels had a worthwhile reduction. Hyperlipidaemia was an important factor in this study : 18 of 19 patients (14 untreated) developing infarction in the first 39 months had a hyperlipidaemia. But no correlation was found between lipidlowering effects and benefit from clofibrate; no infarction occurred in hyperlipidaemic subjects not responding to clofibrate. It is not clear if the benefit from clofibrate is still significant when these non-responders are excluded from consideration. This trial was not double-blind and is open to criticism on the grounds that the treated patients may have become more "coronary conscious" and taken other protective measures. Krasno and Kidera have also made an interim report of a continuing primary prevention study in young males without heart disease. When 1,049 patients left untreated for 23 months are compared with 1,169 patients treated with clofibrate for 32 months a significant difference in the incidence of coronary artery disease is already evident. Further results from this study will be interesting; so too will be the reports from the large doubleblind primary prevention trial at present proceeding in Edinburgh and Budapest.

These studies have not defined the place of clofibrate in clinical practice. Indeed they have cast some doubt on the generally accepted theory that the drug acts by reducing the levels of cholesterol and triglycerides. But it would be premature to dismiss this explanation on the present inconclusive evidence. Clofibrate does lower serum cholesterol and triglyceride levels in most patients, and a reduction in hepatic lipid content has been demonstrated. ${ }^{6} \mathrm{~A}$ block occurs in cholesterol biosynthesis at a stage between acetate and mevalonate, so that precursors prior to the site of the block are readily metabolized, and potentially atherogenic precursors do not accumulate. ${ }^{7}$ Though increased faecal excretion of cholesterol and its metabolites has been suggested as a further mode of action of clofibrate, ${ }^{8}$ recent studies have shown decreased faecal excretion of bile acid, probably reflecting reduced hepatic synthesis and catabolism of cholesterol. ${ }^{9}$ The effect on triglyceride may be a result of the occupation by clofibrate, being strongly protein-bound, of binding sites used for the transport of non-esterified fatty acids, thus reducing their supply to the liver, with a resultant fall in triglyceride synthesis. ${ }^{10}$ But there are other possible mechanisms of action. Clofibrate does have effects on plasma fibrinogen, ${ }^{11}$ platelet adhesiveness, ${ }^{12}$ and on the vascular wall, ${ }^{13}$ and these may play some part in the prevention of atherosclerosis or arterial thrombosis.

There is still a real need for a completely satisfactory, multicentre study of the prevention of further infarction. Only after many more measurements have been made can the mode of action and usefulness of clofibrate be decided. For the moment its use remains empirical. The results at present available give general support to the current practice of prescribing clofibrate for patients with any degree of hyperlipidaemia, especially when there are symptoms of ischaemic heart disease. If the evidence in favour of a nonlipid-lowering mode of action emerges, clofibrate will become widely used for patients without lipid disturbance. But this cannot be recommended at the present time.

\footnotetext{
1 Physicians of the Newcastle upon Tyne Region, British Medical fournal

$1971,4,767$.
2 Research Committee of the Scottish Society of Physicians, British Medical fournal, 1971, 4,775.

3 Gillam, P. M. S., British Medical fournal, 1972, 1, 685.

- Dewar, H. A., and Oliver, M. F., British Medical fournal, 1971 4, 784 .

Krasno, L. R., and Kidera, G. J., fournal of the American Medica Association, 1972, 219, 845 .

Azarnoff, D. L., Tucker, D. R., and Barr, G. A., Metabolism, 1965 14, 959.

Sodhi, H. S., Kudchodkar, B. J., Horlick, L., and Weder, C. H., Metabolism, 1971, 20, 348.

${ }^{8}$ Grundy, S. M., Ahrens, E. H. Jr., Salen, G., and Quintao, E., fournal of Clinical Investigation, $1969,48,33 \mathrm{a}$.

Mitchell, W. D., and L. E. Murchison, Clinica Chimica Acta, 1972

36, 153. 10 Barrett, A. Mritish fournal of Pharmacology, 1966, 26, 363.

11 Cotton, R. C., Wale, F., and Spiller, G. W., fournal of Atherosclerosis Research, 1963, 26, 363.

12 Carson, P., et al., British Heart fournal, 1966, 28, 400.

13 Robertson, A. L., Circulation, 1968, 37 and 38, Suppl. 6, p. 20
}

\section{Carcinoma of the Cervix and Herpesvirus}

The mortality rates for cancer of the cervix uteri have been declining in successive generations of women in England and Wales except for an apparent reversal of the downward trend in the group born between 1911 and $1924 .^{1}$ It had occurred to G. B. Hill and A. M. Adelstein that these women would have been in adolescence and early womanhood during the years of the second world war, when sexual customs were changing and promiscuity, which is known to be associated with carcinoma of the cervix, was increasing. The extension of the study to some 18 other countries required that rates for carcinoma of the uterus be used because there were inadequate data for the cervix alone. ${ }^{2}$ Adelstein, Hill, and $\mathbf{L}$. Maung ${ }^{2}$ found 11 countries where a similar rise in the death rate occurred at about the same time, each followed by a subsequent fall, though two countries, the U.S.A. and Japan, showed no changes in the downward trend over the birth period 1889-1929 - that is, for women aged 40-80 in 1969. They believe that the only explanation for the sudden peak is the prevalence, especially during the second world war, of a venerally transmitted infectious agent such as Herpesvirus hominis type 2 (HSV-2).

If the virus is not the direct cause of this cancer, then the evidence for its association with the disease is either that 
virus infection and cervical anaplasia are independently related to promiscuity, or that HSV-2 may grow preferentially in preneoplastic, or neoplastic, cervical tissue. ${ }^{3}$ Adelstein and his colleagues $^{2}$ have unfortunately no serological data to support their thesis. A. Singer ${ }^{4}$ reports that between one and four years after known infection with HSV-2 $91 \%$ of women had antibodies to the virus. Herpesviruses are persistent agents, and it might be expected therefore that the cohorts in question would have reversals in the trend of HSV-2 antibodies comparable with the mortality rates.

Attempts to isolate the virus itself from cervical lesions have been largely unsuccessful. In a study of 688 males with a history of venereal disease only 29 produced HSV-2.5 Moreover, infectious virus can rarely be found in animal cancers produced by viruses containing deoxyribonucleic acid (DNA). But there is evidence that in common with such viruses HSV-2 will "transform" hamster embryo fibroblasts in vitro" and will give a small yield of tumours after inoculation into newborn hamsters. ${ }^{7}$ Yet so do several adenovirus strains, and evidence that these play any part in human cancers (for example, respiratory system) is lacking.

A. J. Nahmias and colleagues ${ }^{7}$ have reported that HSV antigens can be detected by immunofluorescent techniques in cervical cancer cells in vivo and also in those grown in tissue culture. This is new evidence for the association of HSV-2 with carcinoma of the cervix, but the association may have no bearing on the cause of the cancer. What is needed now is for epidemiologists to study two comparable groups of women-one shown to have been exposed to a genital infection with HSV-2 and the other to differ in that respect only.

In the short term at least one line of investigation could repay research. The oncogenic animal viruses all appear to change the heredity of the cells which they convert to malignancy by adding, and incorporating, virus genetic material (DNA) into that of the normal cell. This additional DNA may be recognized biochemically in the cancer cell's nucleus and may possibly be identifiable in situ histochemically. ${ }^{8}$ Subject to two important provisos this is probably the best way of finding out whether a tumour is of viral origin. The first proviso is that the identification process is specific and that small pieces of normal DNA in the cell's nucleus do not interfere with it. (There is, for instance, recent evidence 9 that "normal" chick cell DNA may contain small pieces of avian tumour virus or virus-like DNA.) The second proviso is similar. It must be shown that only malignancy arises from this integration of viral and cellular DNA. It is possible that latent, or persistent, virus infections are also characterized by the persistence of viral genetic material in close association with the genetic material of the cell. If this proves to be the case for the herpesviruses, then, like the serological and epidemiological evidence, its significance will also be equivocal.

1 Hill, G. B., and Adelstein, A. M., Lancet, 1967, 2, 605.

2 Adelstein. A. M., Hill, G. B., and Maung, L., British fournal of Preventive and Social Medicine, 1971, 25, 186.

3 British Medical Fournal 1970, 4, 256

4 Singer, A., British Medical fournal, 1971, 1, 458.

5 Jeansson, S., and Molin, L., British Medical fournal, 1971, 3, 247.

- Duf, R., and Rapd, F., Fournal of Virology, 1971, 8, 469.

7 Nahmias. A. J., Naib. Z. M.. and Josey, W. E., in Onc.ngenesis and Herpesviruses, ed. P. M. Biggs, G. de The, and Payne, L. N. Herpesviruses, ed. P. M. Biggs, G. de Thé, and Payne, L. N.
Paris. International Agency for Research on Cancer, 1972 (In Press)

8 McDougall, J. K., Dunn, A. R., and Jones, K. W., Nature, 1972 , 236. 346.

- Varmus, H. E., Weiss, R. A., Friis, R. R., Levinson, W., and Bishop, I. M.', Proceedings of the National Academy of Sciences of the United States of America, 1972, 69, 20.

\section{Infection in Rheumatoid Disease}

The seriousness of infection in patients suffering from rheumatoid disease has been recognized since 1953, when its importance as a cause of death was stressed. ${ }^{1}$ That there might be an increased susceptibility to infection has also been suggested in an attempt to explain an apparent increased incidence of bronchiectasis, pneumonia, and possibly bronchitis in people suffering from rheumatoid arthritis. ${ }^{2}$ Similarly, these patients are thought to have an increased incidence of urinary infections and chronic pyelonephritis, but the interpretation of the findings is questionable because of the high ingestion of analgesics.

In 1958 attention was drawn to the problems of suppurative arthritis, often polyarticular, bone infection, and skin abscesses occuring in 12 patients with severe rheumatoid arthritis. ${ }^{4}$ Increase in joint pain and swelling in several joints, together with deterioration in general health, were first attributed to an exacerbation of the rheumatoid process. In two cases the correct diagnosis was made only when a swelling discharged pus and in three at necropsy. The most common infecting agent was a staphylococcus. Fever and leucocytosis did not always occur. The serious nature of these infections is clear from the fact that five of the first seven patients died, infection having not been suspected until too late for adequate treatment.

An increase in awareness of this problem has led to better diagnosis, but it is still not always recognized early. In a recent series of 17 such patients the mean duration of onset of symptoms to diagnosis was nine days, compared with four days in uncomplicated septic arthritis. In three there was a delay of two weeks. 5 Two of these three proved difficult to treat, suffering recurrences despite adequate antibiotic therapy initially. Staphylococcus aureus, often penicillin-resistant, was the most usual infecting organism, and in 9 of the 17 cases the same organism was cultured from swabs of ulcerated areas and such infections as a paronychia. The multiplicity of joints involved and the frequency of positive blood cultures indicates that septicaemia or bacteraemia from such sites is common. Great care should therefore be taken in the management of all ulcerated areas in any patient suffering from severe seropositive rheumatoid arthritis.

But infection is not confined to the skin, bones, and joints. Twelve patients with seropositive rheumatoid arthritis, six of whom were on corticosteroids, suffered a total of 24 infections. 6 These included septicaemic endocarditis and abscesses in many sites, including muscular areas such as the calf and thigh, ovary, pelvis, and perinephric region. Infection in the lung, sometimes leading to lung abscesses as well as empyema, was common, while suppurative pericarditis was also seen. These infections might or might not be associated with pyoarthroses and were often multiple. Of particular note was the fact that recurrent infections not necessarily with the same organism occurred at different sites in five patients. Death resulted from three of the 24 infections.

The frequency of infection in Felty's syndrome is well known. ${ }^{7}$ However, only one of these patients was considered to be suffering from this. Similarly the administration of corticotrophin and corticosteroids, particularly in high dosage, is known to predispose to infection. ${ }^{8}$ Yet in this last series only six patients were receiving steroids. 